\title{
Hemophagocytic lymphohistiocytosis in adults
}

\author{
Yadav Pandey, MD ${ }^{\mathrm{a}}$ (D), Dinesh Atwal, MD ${ }^{\mathrm{a}}$, Manojna Konda, $\mathrm{MD}^{\mathrm{a}}$ (D), Milan Bimali, $\mathrm{PhD}^{\mathrm{b}}$, Derek Middleton, $\mathrm{MD}^{\mathrm{a}}$, \\ Naveen Yarlagadda, $\mathrm{MD}^{\mathrm{a}}$, Belal Firwana, $\mathrm{MD}^{\mathrm{C}}$, and Appalanaidu Sasapu, MDa \\ ${ }^{a}$ Department of Hematology and Oncology, Winthrop P. Rockefeller Cancer Institute, University of Arkansas for Medical Sciences, Little Rock,

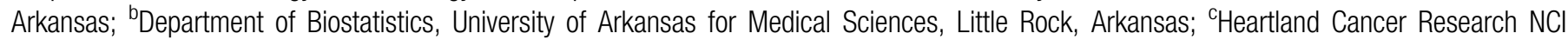 \\ Community Oncology Research Program, Missouri Baptist Medical Center, St. Louis, Missouri
}

\begin{abstract}
Hemophagocytic lymphohistiocytosis $(\mathrm{HLH})$ is an underrecognized disorder due to the variability of its presentation and the fact that in adults, its diagnosis is based on cumbersome, pediatric-based criteria. Data regarding demographics, underlying causes, clinical features, laboratory results, complications, treatments received, and clinical outcomes were collected and analyzed in 41 patients who were diagnosed and treated at University of Arkansas for Medical Sciences between 2007 and 2019. In this group, $51 \%$ were male, the median age at diagnosis was 47 years, and 85\% (35/41) met the HLH-2004 diagnostic criteria (5/8 variables). When evaluating seven extended variables easily obtained by routine laboratory test, $93 \%$ (38/41) of patients met 8 out of 15 criteria. The overall mortality in our patient population was $54 \%(22 / 41)$. The 30 -day and 1 -year overall survival estimates were $0.73(95 \%$ confidence interval $0.56,0.84)$ and $0.46(95 \%$ confidence interval $0.29,0.62)$, respectively. Thirty-five patients (85.4\%) received HLH-directed therapy, and 19 patients (46.3\%) achieved remission. The most common regimen for treating HLH was dexamethasone plus etoposide (53.7\%). The patients with malignancy-related HLH had a worse prognosis than those without underlying malignancy, with a $73.33 \%(11 / 15)$ vs $34.62 \%(9 / 26)$ mortality $(P=0.02)$. In conclusion, despite increasing recognition, HLH remains an enigmatic disorder with increased mortality, even more so with malignancy-associated HLH.
\end{abstract}

KEYWORDS Cytopenia; hemophagocytic lymphohistiocytosis; hemophagocytosis

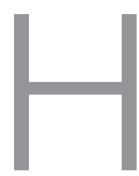

emophagocytic lymphohistiocytosis (HLH) is an underrecognized and poorly understood disorder characterized by uncontrolled stimulation of the immune system that runs amok and results in end-organ damage which can be life threatening. ${ }^{1,2}$ HLH can be either a primary process due to the genetic mutations affecting cytotoxic $\mathrm{T}$ cells or natural killer (NK) cells ${ }^{3}$ or can be acquired (secondary) due to underlying malignancy, infection, rheumatologic disorder, or immune deficiency., Although initially described in the pediatric population, there has been a dramatic increase in the reported cases of HLH in adults over the past decade. ${ }^{5}$ Despite increasing recognition, much of our current knowledge of HLH in adults is based on the experiences and data on pediatric HLH. The studies on HLH in the adult population are very few and largely retrospective. ${ }^{6,7}$ Despite several propositions in the past few years to establish diagnostic criteria specific for HLH in adults, ${ }^{8,9}$ we currently continue to follow the
Histiocyte Society's HLH-2004 pediatric diagnostic criteria, which were derived solely for the pediatric population. ${ }^{10}$ Hence, there is a need for further studies in adults with HLH to help clarify diagnostic criteria and improve outcomes. We sought to examine the clinical characteristics, underlying triggers, complications, management, and outcomes of adult patients diagnosed and treated in our institute and to compare our experience with that in the literature with an aim to increase overall knowledge of this highly fatal disease.

\section{METHODS}

This single-center retrospective study was performed at the University of Arkansas for Medical Sciences. Between 2007 and 2019, we identified 46 adult patients who were diagnosed and treated for HLH in our institution. Five patients with insufficient data were excluded from the study. For the remaining 41 patients, data regarding demographics,

Corresponding author: Yadav Pandey, MD, Department of Hematology and Oncology, Winthrop P. Rockefeller Cancer Institute, University of Arkansas for Medical Sciences, 4301 W Markham, Slot \#634, Little Rock, AR 72205 (e-mail: ypandey@uams.edu)

Received January 21, 2020; Revised February 22, 2020; Accepted February 27, 2020. 
Table 1. Clinical characteristics and underlying triggers for 41 adults with hemophagocytic lymphohistiocytosis

\begin{tabular}{|c|c|c|}
\hline Category & Variable & $n(\%)$ \\
\hline \multirow[t]{2}{*}{ Age at diagnosis } & Mean (SD) (years) & $48(19)$ \\
\hline & Median (range) (years) & $47(15-78)$ \\
\hline \multirow[t]{2}{*}{ Sex } & Male & $21(51 \%)$ \\
\hline & Female & $20(49 \%)$ \\
\hline \multirow[t]{3}{*}{ Ethnicity } & White & $30(73 \%)$ \\
\hline & Black & $8(20 \%)$ \\
\hline & Other & $3(7 \%)$ \\
\hline \multirow[t]{13}{*}{ Underlying condition } & Infection & $31(76 \%)$ \\
\hline & Epstein-Barr virus & $9(29 \%)$ \\
\hline & Cytomegalovirus & $5(16 \%)$ \\
\hline & HIV with disseminated Histoplasma & $5(16 \%)$ \\
\hline & Histoplasma in non-HIV & $3(10 \%)$ \\
\hline & Cancer & $15(37 \%)$ \\
\hline & Non-Hodgkin's Iymphoma & $7(47 \%)$ \\
\hline & Multiple myeloma & $3(20 \%)$ \\
\hline & Hodgkin's lymphoma & $1(7 \%)$ \\
\hline & Breast cancer & $1(7 \%)$ \\
\hline & Rheumatologic disorder & $9(22 \%)$ \\
\hline & Systemic lupus erythematosus & $5(56 \%)$ \\
\hline & Rheumatoid arthritis & $2(22 \%)$ \\
\hline
\end{tabular}

underlying causes, clinical features, laboratory results, complications, treatment, and outcome were collected and analyzed. Based on the HLH-2004 criteria, data on eight variables-fever, splenomegaly, cytopenia, hypertriglyceridemia, hyperfibrinogenemia, elevated ferritin, presence of hemophagocytosis on biopsy, elevated soluble IL-2 receptor level, and low/absent NK cell activity-were collected.

We also collected data on seven additional variables easily obtained on routine laboratory or physical examination, which have been suggested by experts as useful variables in diagnosing HLH. ${ }^{11,12}$ Those variables include the presence of hepatitis (defined as liver enzymes 2.5 times the upper limit of normal), hepatomegaly (clinical exam or imaging), elevated lactate dehydrogenase (>200 IU/L), coagulopathy (defined as prothrombin time $\geq 1.5$ times normal, activated partial thromboplastin time $\geq 1.5$ times normal, or D-dimer $>10 \mathrm{mcg} / \mathrm{mL}$ ), monocytosis (absolute monocyte count $>1.0$ $\left.\times 10^{9} / \mathrm{L}\right)$, hypoalbuminemia $(<3.5 \mathrm{~g} / \mathrm{dL})$, and renal failure ( $\geq 50 \%$ increase in serum creatinine). Some experts also suggest beta- 2 microglobulin level as a useful variable for diagnosis of HLH. ${ }^{13}$ As we had data on only five patients' beta-2 microglobulin levels, we did not include it in our results.

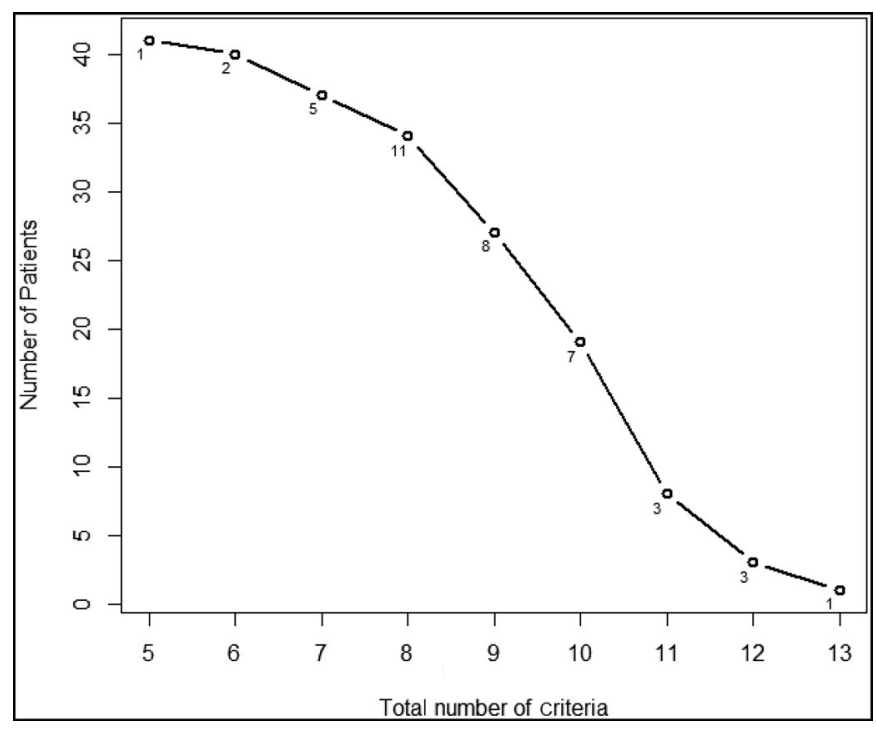

Figure 1. Plot of number of criteria vs patients. The plot represents the number of patients ( $y$ axis) against $\geq$ total number of criteria. The numbers within the plot represents the number of patients who experienced the exact number of criteria.

Combining eight HLH-2004 criteria and seven additional variables, we plotted the number of patients meeting each criterion in a graph.

Descriptive statistics were reported as mean (standard deviation) and median (interquartile range) for continuous variables, and as frequency (percentage) for categorical variables. The survival time was calculated as the difference between the date of diagnosis and date of death (for patients who died) or the date of last follow-up (for patients who did not experience death and were censored). The date of death was not available for two patients. For these patients, the date of their last follow-up was used as a conservative approach to estimate the date of death. The distribution of overall survival was presented using Kaplan-Meier survival curve. The overall survival at 30 days and 365 days was estimated using Kaplan-Meier curve. Bivariate association was assessed using a chi-square test. A two-sided $P$ value of 0.05 was used to determine statistical significance. The analysis was done in SAS 9.4 and R 3.6.1.

\section{RESULTS}

The median age at diagnosis was 47 years (range 15-78). There were 21 men $(51 \%)$ and 20 women (49\%), with most of the patient population being white $(73 \%)$. For underlying etiologies, 31 patients $(76 \%)$ had underlying infection, the most common of which was Epstein-Barr virus (29\%); 15 patients (37\%) had underlying malignancy, with nonHodgkin's lymphoma being the most common malignancy (47\%); and 9 patients (22\%) had an underlying rheumatologic disorder (Table 1).

Among the variables in HLH-2004 criteria, fever, cytopenia, and elevated ferritin were the most common, with 40 patients $(97.6 \%)$ meeting the criteria. Among the remaining 
Table 2. Different criteria met by 41 adults with hemophagocytic lymphohistiocytosis

\begin{tabular}{llr}
\hline Group & \multicolumn{1}{c}{ Criterion } & $\boldsymbol{n}(\%)$ \\
\hline HLH-2004 criteria & Fever & $40(98 \%)$ \\
& Splenomegaly & $17(41 \%)$ \\
& Cytopenia & $40(98 \%)$ \\
& Hypertriglyceridemia & $24(59 \%)$ \\
& Hypofibrinogenemia & $18(44 \%)$ \\
& Elevated ferritin & $40(98 \%)$ \\
& Hemophagocytosis (N=35) & $28(80 \%)$ \\
& Increased IL-2 receptors $(N=27)$ & $24(89 \%)$ \\
& Low/absent NK cell activity $(N=8)$ & $7(88 \%)$ \\
Patients meeting 5 of 8 HLH-2004 criteria & $35(85 \%)$ \\
Extended variables & Hepatitis & $26(63 \%)$ \\
& Elevated lactatedehydrogenase & $38(93 \%)$ \\
& Hepatomegaly & $6(15 \%)$ \\
Catients meeting 8 out of 15 total criteria & $29(71 \%)$ \\
\hline & Coagulopathy & $10(24 \%)$ \\
Monocytosis & $29(71 \%)$ \\
Acute kidney failure & $38(93 \%)$ \\
& Hypoalbuminemia & $38(93 \%)$ \\
\hline
\end{tabular}

criteria, 27 patients were tested for increased IL-2 receptors, and 24 tested positive (89\%). Only 8 were tested for NK cell activity, out of which 7 had low/absent NK cell activity $(88 \%)$. Of the 35 patients who underwent biopsy, 28 (80\%) had hemophagocytosis. Overall, 85\% (35/41 patients) met the HLH-2004 diagnostic criteria (5/8 criteria). We also evaluated for the seven extended variables described earlier. Out of 41 patients, 38 patients (93\%) had elevated lactate dehydrogenase and hypoalbuminemia; 26 patients (63\%) met the criteria for hepatitis; 29 patients (71\%) met criteria for coagulopathy and acute kidney injury; 6 patients (15\%) had hepatomegaly; and 10 patients (24\%) had monocytosis. Combining the HLH-2004 criteria with extended variables, we plotted the number of patients meeting the number of criteria out of a total of 15 (Figure 1). A total of 38 patients out of $41(93 \%)$ met 8 out of 15 criteria (Table 2).

The overall mortality in our population was 54\% (22 out of 41). The 30-day and 1-year overall survival estimate was $0.73(95 \%$ confidence interval [CI]: $0.56,0.84)$ and 0.46 (95\% CI: 0.29, 0.62\%). Nineteen patients (46.3\%) achieved remission, with $3(7 \%)$ relapsing later despite treatment. Approximately 35 patients (85.4\%) received HLH-targeted therapy, and 32 patients $(78 \%)$ received treatment for their underlying illness as well. Nine of 15 patients $(60 \%)$ received treatment for underlying malignancy. The most common

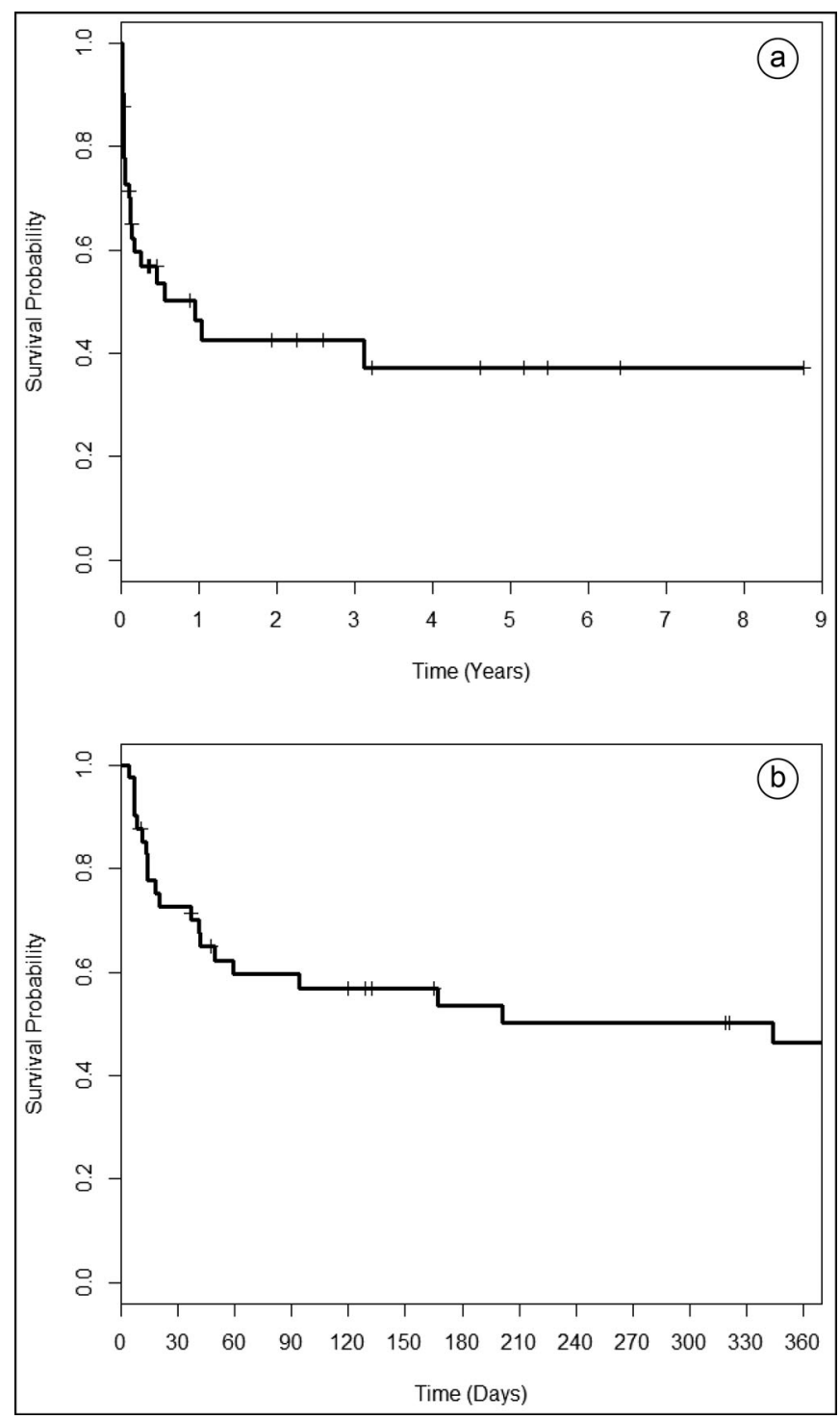

Figure 2. (a) Distribution of overall survival. (b) Distribution of overall survival restricted to 365 days.

regimen for treating HLH was dexamethasone and etoposide, with 22 patients $(53.7 \%)$ receiving this regimen during their illness. Five of 35 patients (14\%) received high-dose dexamethasone alone. The patients with malignancy had a worse prognosis than patients without malignancy: $73.33 \%$ $(11 / 15)$ vs $34.62 \%(9 / 26)$ death $(P=0.02)$. Figure 2 shows the Kaplan-Meier survival curve.

\section{DISCUSSION}

Adult HLH is a rare and devastating disorder of a hyperactive immune system. If not recognized in a timely manner, it has a dismal prognosis, with a median survival of $<2$ months if left untreated. ${ }^{14}$ It often eludes diagnosis on presentation, causing a delay, as its clinical features are nonspecific and frequently masquerade as the underlying processes including infection, malignancy, and rheumatologic disease.

While there are limited data on the incidence of HLH in adults, a systematic review of 2197 adult cases worldwide by 
Ramos-Casals et al reported a female preponderance (1:7) with a mean age at diagnosis of 50 years. ${ }^{15}$ However, in our patient population, we found that the incidence in males and females was almost the same, with a mean age of diagnosis of 47 years.

A meticulous search for the cause of HLH in adults is warranted, as it is frequently incited by an underlying disease process. A retrospective analysis of 162 adults with $\mathrm{HLH}$ reported malignancy (60\%; with B-cell lymphoma, $22 \%$, as the most common malignancy), infections (25\%), and autoimmune conditions (3\%) as the associated conditions. ${ }^{16}$ Ramos-Casals et al reported that infections were the most common triggers in adult HLH (50.4\%) followed by malignancy $(47.6 \%)$ and autoimmune diseases, with systemic lupus erythematosus the most commonly reported autoimmune disorder. ${ }^{15}$ In our institutional study, HLH primarily arose in the setting of infection, which was noted in $76 \%$ of the cases. Epstein-Barr virus was the most commonly associated infection, followed by HIV with an associated histoplasmosis infection and cytomegalovirus infection. This is likely a reflection of local epidemiology, as Arkansas is known for its concentration of endemic fungal infections. While underlying malignancy was noted in $37 \%$, it was not surprising that multiple myeloma was the second most common malignancy in our population, since our renowned myeloma institute has a sizeable patient population.

Current understanding of the diagnosis and management of adult HLH continue to depend heavily on our experiences and data from pediatric HLH. Several limitations are recognized in applying the pediatric HLH-2004 diagnostic criteria to adults. ${ }^{11,17}$ These diagnostic criteria also have other pitfalls, as some are nonspecific and can be a manifestation of the underlying malignancy, infection, rheumatologic problem, or immunodeficiency, while tests for IL-2 receptors and NK cell activity are not readily available at all centers and results can take weeks. In our patient population, 33 patients $(81 \%)$ were not tested for NK cell activity and 14 patients (34\%) were not tested for soluble IL-2 receptor. Most of our patients $(85 \%)$ underwent a bone marrow biopsy. Despite the diagnostic difficulties encountered, 85\% (35/41) of our patient population met the criteria for HLH.

The management of HLH predominantly focuses on treating the underlying cause and controlling the hyperstimulated immune system. Treatment in adults is guided by the HLH-94 study, which was a prospective study in a pediatric population with no history of immunosuppression or malignancy. This study demonstrated that 8-week induction with dexamethasone and etoposide showed significant improvement in survival. ${ }^{18}$ Arca et al conducted a retrospective analysis in 162 adults and found that use of etoposide was associated with better outcomes. ${ }^{6}$ However, a study in 62 adults by Parikh et al found that the overall survival did not statistically vary between etoposide- and nonetoposidereceiving cohorts. ${ }^{19}$ In patients with aggressive lymphomas, doxorubicin-based therapy such as cyclophosphamide, doxorubicin, vincristine, and prednisone (CHOP) has also been used. In a small retrospective analysis, use of the CHOP regimen in adults with HLH due to lymphoma or Epstein-Barr virus showed an overall response rate of $58.8 \% .{ }^{20}$ While allogenic hematopoietic stem cell transplant is the mainstay of treatment in pediatric patients with HLH, it is also being used in adults with a known genetic predisposition or with recurrent $\mathrm{HLH}^{21}$

In our study, $83 \%$ of patients were treated for HLH and $74 \%$ also received treatment for their underlying HLH triggers. The most common regimen used in our patients was the combination of dexamethasone and etoposide, followed by high-dose dexamethasone alone. Approximately 15\% of patients did not receive HLH-targeted therapy. These patients were either diagnosed late and care was focused toward comfort or were clinically stable and treated only for underlying triggers. Recently, the Food and Drug Administration approved emapalumab, an anti-IFN- $\gamma$ monoclonal antibody, for the treatment of pediatric and adult patients with primary HLH with refractory disease, and initial results have been promising. ${ }^{22}$ Also, there is an emerging role of the JAK inhibitor ruxolitinib in refractory secondary HLH. ${ }^{22}$ The long-term efficacy of these drugs is yet to be established.

The overall mortality for our patient population remained high (45\%) despite $83 \%$ (33/41) receiving treatment. A systematic scoping review by Hayden et al has shed further light on the heterogeneity of this disorder. This review article described a mortality rate varying from $20.4 \%$ to as high as $88 \%$ depending on the population factors and length of follow-up of the comprising studies. ${ }^{7}$ The patients with malignancy-associated HLH have a worse prognosis than those with nonmalignancy-associated $\mathrm{HLH}$, with a median overall survival of 1.4 months and 22.8 months, respectively. ${ }^{19}$ This is comparable with the significantly higher mortality seen in the malignancy-associated HLH group in our study (mortality $73.33 \%$ vs $34.62 \%, P=$ 0.02 ). The major limitations of our study are the retrospective model of the study, relatively small sample size, singlecenter population, and case identification dependent on medical record diagnosis coding.

In conclusion, increasing awareness of adult HLH helps clinicians identify the disease process earlier in the course and initiate prompt treatment to help reduce mortality. There is still an unmet need to refine the diagnostic criteria. Due to relative rarity and dismal prognosis, multicenter studies especially targeting patients with malignancy-associated $\mathrm{HLH}$ are urgently needed to further improve the understanding of this syndrome.

\section{ORCID}

Yadav Pandey (D) http://orcid.org/0000-0003-2307-7310 Manojna Konda (D) http://orcid.org/0000-0001-8631-741X

1. Filipovich A, McClain K, Grom A. Histiocytic disorders: recent insights into pathophysiology and practical guidelines. Biol Blood 
Marrow Transplant. 2010;16(1):S82-S89. doi:10.1016/j.bbmt.2009. 11.014 .

2. George MR. Hemophagocytic lymphohistiocytosis: review of etiologies and management. J Blood Med. 2014;5:69-86. doi:10.2147/JBM. S46255.

3. Jordan MB, Allen CE, Weitzman S, Filipovich AH, McClain KL. How I treat hemophagocytic lymphohistiocytosis. Blood. 2011; 118(15):4041-4052. doi:10.1182/blood-2011-03-278127.

4. Machaczka M, Vaktnas J, Klimkowska M, Hagglund H. Malignancyassociated hemophagocytic lymphohistiocytosis in adults: a retrospective population-based analysis from a single center. Leuk Lymphoma. 2011;52(4):613-619. doi:10.3109/10428194.2010.551153.

5. Clementi R, Emmi L, Maccario R, et al. Adult onset and atypical presentation of hemophagocytic lymphohistiocytosis in siblings carrying PRF1 mutations. Blood. 2002;100(6):2266-2267. doi:10.1182/ blood-2002-04-1030.

6. Arca M, Fardet L, Galicier L, et al. Prognostic factors of early death in a cohort of 162 adult haemophagocytic syndrome: impact of triggering disease and early treatment with etoposide. $\mathrm{Br} J$ Haematol. 2015;168(1):63-68. doi:10.1111/bjh.13102.

7. Hayden A, Park S, Giustini D, Lee AYY, Chen L. Hemophagocytic syndromes (HPSs) including hemophagocytic lymphohistiocytosis (HLH) in adults: A systematic scoping review. Blood Rev. 2016;30(6): 411-420. doi:10.1016/j.blre.2016.05.001.

8. Fardet L, Galicier L, Lambotte O, et al. Development and validation of the HScore, a score for the diagnosis of reactive hemophagocytic syndrome. Arthritis Rheumatol (Hoboken, NJ). 2014;66(9):2613-2620. doi:10.1002/art.38690.

9. Hejblum G, Lambotte O, Galicier L, et al. A web-based Delphi study for eliciting helpful criteria in the positive diagnosis of hemophagocytic syndrome in adult patients. PLoS One. 2014;9(4):e94024-e94024. doi:10.1371/journal.pone.0094024.

10. Henter J-I, Horne A, Arico M, et al. HLH-2004: Diagnostic and therapeutic guidelines for hemophagocytic lymphohistiocytosis. Pediatr Blood Cancer. 2007;48(2):124-131. doi:10.1002/pbc.21039.

11. Tamamyan GN, Kantarjian HM, Ning J, et al. Malignancy-associated hemophagocytic lymphohistiocytosis in adults: relation to hemophagocytosis, characteristics, and outcomes. Cancer. 2016;122(18): 2857-2866. doi:10.1002/cncr.30084.
12. Schram AM, Campigotto F, Mullally A, et al. Marked hyperferritinemia does not predict for HLH in the adult population. Blood. 2015; 125(10):1548-1552. doi:10.1182/blood-2014-10-602607.

13. Hibi S, Ikushima S, Fujiwara F, et al. Serum and urine beta-2-microglobulin in hemophagocytic syndrome. Cancer. 1995;75(7):1700-1705. doi:10. 1002/1097-0142(19950401)75:7<1700::AID-CNCR2820750722>3. $0 . \mathrm{CO} ; 2-\mathrm{D}$.

14. Janka G. Hemophagocytic lymphohistiocytosis: when the immune system runs amok. Klin Padiatr. 2009;221(05):278-285. doi:10.1055/ s-0029-1237386.

15. Ramos-Casals M, Brito-Zeron P, Lopez-Guillermo A, Khamashta MA, Bosch X. Adult haemophagocytic syndrome. Lancet. 2014; 383(9927):1503-1516. doi:10.1016/S0140-6736(13)61048-X.

16. Riviere S, Galicier L, Coppo P, et al. Reactive hemophagocytic syndrome in adults: a retrospective analysis of 162 patients. Am J Med. 2014;127(11):1118-1125. doi:10.1016/j.amjmed.2014.04.034.

17. Zhang Z, Wang J, Ji B, et al. Clinical presentation of hemophagocytic lymphohistiocytosis in adults is less typical than in children. Clinics. 2016;71(4):205-209. doi:10.6061/clinics/2016(04)05.

18. Henter JI, Arico M, Egeler RM, et al. HLH-94: a treatment protocol for hemophagocytic lymphohistiocytosis. HLH Study Group of the Histiocyte Society. Med Pediatr Oncol. 1997;28(5):342-247. doi:10.1002/(SICI)1096-911X(199705)28:5<342::AID-MPO3>3.0. $\mathrm{CO} ; 2-\mathrm{H}$.

19. Parikh SA, Kapoor P, Letendre L, Kumar S, Wolanskyj AP. Prognostic factors and outcomes of adults with hemophagocytic lymphohistiocytosis. Mayo Clin Proc. 2014;89(4):484-492. doi:10.1016/j. mayocp.2013.12.012.

20. Shin H-J, Chung JS, Lee J-J, et al. Treatment outcomes with CHOP chemotherapy in adult patients with hemophagocytic lymphohistiocytosis. J Korean Med Sci. 2008;23(3):439-444. doi:10.3346/jkms.2008. 23.3.439.

21. Fu L, Wang J, Wei N, et al. Allogeneic hematopoietic stem-cell transplantation for adult and adolescent hemophagocytic lymphohistiocytosis: a single center analysis. Int J Hematol. 2016;104(5):628-635. doi: 10.1007/s12185-016-2062-7.

22. Lounder DT, Bin Q, de Min C, Jordan MB. Treatment of refractory hemophagocytic lymphohistiocytosis with emapalumab despite severe concurrent infections. Blood Adv. 2019;3(1):47-50. doi:10.1182/ bloodadvances.2018025858. 